\title{
Effect of Cannabidiol on Interictal Epileptiform Activity and Sleep Architecture in Children with Intractable Epilepsy: A Prospective Open-Label Study
}

\author{
Kerstin A. Klotz ${ }^{1,2,3} \cdot$ Daniel Grob ${ }^{1,3}$. Jan Schönberger ${ }^{1,2,3} \cdot$ Lea Nakamura ${ }^{1,3} \cdot$ Birgitta Metternich $^{1}$. \\ Andreas Schulze-Bonhage ${ }^{1}$. Julia Jacobs ${ }^{3,4}$
}

Accepted: 30 September 2021 / Published online: 22 October 2021

(c) The Author(s) 2021

\begin{abstract}
Background Cannabidiol has been shown to be effective in seizure reduction in patients with Dravet syndrome, LennoxGastaut syndrome, and tuberous sclerosis. However, very little is known about its potential to reduce interictal epileptiform activity and improve sleep architecture.

Objective The objective of this prospective study was to evaluate the influence of cannabidiol therapy on the frequency of interictal epileptiform discharges (IEDs) and sleep microstructure in a cohort of children with drug-resistant epilepsy.

Methods Children with drug-resistant epilepsy were prospectively followed from November 2019 to January 2021 during an open-label trial of cannabidiol at a dose of $20 \mathrm{mg} / \mathrm{kg} /$ day (to a maximum of $50 \mathrm{mg} / \mathrm{kg} /$ day) and stable concomitant medication. Electroencephalograms were recorded at baseline (T0) and after 3 months (T1). Two independent raters, blinded to clinical outcome, evaluated 5-min segments of sleep stage 2 or low-noise awake state. IEDs were visually identified and rates per minute calculated. Sleep microstructure was considered improved if sleep structures were seen at T1 that were not present at $\mathrm{T} 0$. IED rates at $\mathrm{T} 0$ and $\mathrm{T} 1$ were compared and correlated with seizure outcome, cannabidiol dose, initial IED rate, and disease duration.

Results In total, 35 children (mean \pm standard deviation age $10.1 \pm 0.86$ ) were included. The IED rate at T1 was significantly lower than at T0 $(19.6 \pm 19.5$ vs. $36.8 \pm 27.2$, respectively; $p<0.0001)$. We found a moderate correlation between IED reduction and percentage of seizure reduction compared with baseline (Pearson's $r=0.39 ; p=0.02$ ), a moderate negative correlation between IED reduction and IED rate at T0 $(r=-0.34 ; p=0.04)$, and a trend towards a moderate negative correlation between IED reduction and disease duration $(r=-0.32 ; p=0.06)$. Sleep was recorded in 23 patients. Sleep microstructure was initially abnormal in $56.5 \%$ of sleep recordings and improved in $84.6 \%$ of those cases.

Conclusion Our results strongly suggest the utility of cannabidiol in reducing IEDs and improving sleep microstructure in children with drug-resistant epilepsy. Larger controlled studies are needed to evaluate the clinical relevance of this effect in different epilepsy types.
\end{abstract}

Trial Registration DRKS00013177; 25 June 2019.

\section{Introduction}

The use of cannabidiol in epilepsy treatment has increased markedly over the last 5 years. Four randomized controlled

Kerstin A. Klotz

kerstin.alexandra.klotz@uniklinik-freiburg.de

1 Faculty of Medicine, Freiburg Epilepsy Center, Medical Center-University of Freiburg, University of Freiburg, Freiburg, Germany

2 Berta-Ottenstein-Program, Faculty of Medicine, University of Freiburg, Freiburg, Germany trials showed the efficacy of a plant-derived pharmaceutical grade cannabidiol (Epidiolex ${ }^{\circledR}$; Greenwich Biosciences, Inc., Carlsbad, CA, USA) in patients with Dravet syndrome, Lennox-Gastaut syndrome, and tuberous sclerosis complex [1-4]. Open-label studies have indicated its efficacy in other

3 Department of Neuropediatrics and Muscle Disorders, Faculty of Medicine, Center for Pediatrics, Medical Center-University of Freiburg, University of Freiburg, Mathildenstr. 1, 79106 Freiburg, Germany

4 Section of Pediatric Neurology, Alberta Children's Hospital, Alberta Children's Research Institute, Hodgekiss Brain Institute, University of Calgary, Alberta, Canada 


\section{Key Points}

Cannabidiol has been shown to be effective in reduction of different seizure types, but no studies have evaluated the effect of cannabidiol on interictal epileptiform activity and sleep structures.

This prospective study showed that interictal epileptiform activity reduced significantly and sleep microstructure improved during cannabidiol treatment in children with drug-resistant epilepsy.

Based on the results of this study, it could be hypothesized that reductions in interictal epileptiform discharges and sleep improvement might contribute to potential positive effects of cannabidiol on cognitive functioning and behavior.

types of epilepsy, but evidence is much more limited in these cases [5-7].

The effect of cannabidiol on interictal epileptiform activity is less well-known. Most preclinical in vivo studies used acute seizure models and focused on seizure reduction. Studies using a model of chronic epilepsy and evaluating interictal electroencephalograms (EEGs) are much less common. However, in a study of cannabidiol treatment in a rat model for genetic absence epilepsy (GAERS [Genetic Absence Epilepsy Rats from Strasbourg]), interictal epileptiform discharges (IEDs) decreased significantly after injection of cannabidiol in a similar dose to human treatment [8]. In patients with epilepsy, information on the effect of cannabidiol on IEDs is equally sparse. Evidence is limited to case reports and a few smaller studies with the main focus on seizure reduction but evaluation of EEGs as a secondary objective [9-11]. Similarly, little is known about the effect of cannabidiol on sleep architecture. Although some studies reported a subjective positive effect of cannabidiol on sleep quality [12], to the best of our knowledge, no publications have reported the effect of cannabidiol on sleep architecture in EEG sleep recordings.

Although whether IEDs can be used as a surrogate marker for treatment response in all epilepsies is under debate, they have been shown to be associated with seizure frequency and severity $[13,14]$. Several studies have also shown a correlation between IEDs and neuropsychological disturbances [15, 16]. In some patients, for example, the treatment goal might even be improvement of interictal EEG rather than reduction of seizures [17]. In addition, it is widely accepted that sleep is relevant for memory consolidation and other cognitive processes and that disruption of sleep micro- and macrostructure in patients with epilepsy can contribute to reduced cognitive function $[18,19]$. Therefore, it is important to estimate the effect of antiseizure medication (ASM) on interictal EEG and on sleep architecture. The objective of this prospective study was to evaluate the influence of cannabidiol therapy on interictal epileptiform activity and sleep architecture in a cohort of children with drug-resistant epilepsy.

\section{Methods}

\subsection{Patient Selection}

Children with drug-resistant epilepsy participating in an open-label trial with cannabidiol were prospectively followed. Patient selection criteria included age 3 months to 18 years, pharmacoresistant epilepsy as defined by the International League Against Epilepsy, at least one ASM at a stable dose for 4 weeks preintervention, stable ketogenic diet/vagal nerve stimulation device settings for at least 4 weeks preintervention, and willingness of patients/caregivers to comply with seizure diary. Exclusion criteria were current treatment with cannabis-based products, pregnancy, or unstable hepatic or renal disease [6]. The trial was approved by the institutional research ethics board (397/17) and registered (DRKS00013177; registration date 25 June 2019). All patients or parents/legal representatives provided written informed consent and assent according to patients' physical and mental capability before trial onset.

\subsection{Cannabidiol Treatment}

Patients received either Epidyolex ${ }^{\circledR}$ or a pharmaceutical formulation of synthetic cannabidiol, manufactured by THC Pharm GmbH/Germany, in a $100 \mathrm{mg}$ per mL medium-chain triglyceride oil-based oral solution, according to national drug-preparation regulations. Of the latter, an internal quality control at final solution level was performed at our center. Cannabidiol was administered orally in addition to the baseline antiepileptic drug regimen at a starting dose of $5 \mathrm{mg} / \mathrm{kg} /$ day divided into two daily doses. Patients were advised to take cannabidiol with fatty meals. Dosage was uptitrated by $2-5 \mathrm{mg} / \mathrm{kg} /$ day up to $18-20 \mathrm{mg} / \mathrm{kg} /$ day over approximately 14 days. If seizure reduction was not sufficient, dosage could be increased further up to $50 \mathrm{mg} / \mathrm{kg} /$ day. Efforts were made to keep concomitant doses of antiepileptic drug constant. However, if the addition of cannabidiol led to relevant increases in the serum levels of concomitant ASMs, those antiepileptic drugs were decreased as clinically indicated.

Seizure frequency was recorded for all patients based on a prospective paper diary during a baseline of 4 weeks and during treatment. Parents were asked to document all visible seizures; series of spasms were documented as one seizure. Motor seizures were defined as either focal or generalized seizures with a clear motor component of $\geq 3 \mathrm{~s}$. Mean monthly seizure frequency was identified for baseline period 
(T0) and after 3 months (T1), then the percentage reduction at $\mathrm{T} 1$ compared with $\mathrm{T} 0$ was calculated [6]. Responders were defined as patients with a reduction of $\geq 50 \%$ of their motor seizures.

\subsection{Electroencephalogram (EEG) Acquisition}

All patients underwent resting EEG or video-EEG (VEEG) monitoring prior to cannabidiol treatment and after 3 months of treatment. All EEGs were recorded based on the 10- to 20-electrode placement system according to the relevant clinical protocol. Minimum recording time was according to EEG type (20 minutes for routine EEG when the patient was awake and 30 minutes when the patient was asleep; 24 $h$ for VEEG).

\subsection{EEG Rating}

For this study, only patients for whom the same type of EEG study (either overnight VEEG recordings, daytime recordings awake, or daytime recordings asleep) at $\mathrm{T} 0$ and $\mathrm{T} 1$ were available were included in the analysis. EEGs were visually analyzed independently by two raters. Both raters were blinded to any clinical information, including dose of cannabidiol, change of seizure frequency at $\mathrm{T} 1$, and sequence of EEG recordings. Interrater reliability was calculated using Cohen's kappa. Disagreements were resolved after discussion. The first reader identified and marked the first segment fulfilling the following criteria: (1) at least 10 minutes of uninterrupted sleep with the occurrence of sleep spindles (SS) as an indicator of non-rapid eye movement sleep stage 2 (in patients without physiological sleep architecture, we used an interval without movement artifacts that occurred after the technician's note that the patient had fallen asleep) and (2) at least $1 \mathrm{~h}$ after or before a seizure. If no sleep segment was available, segments of awake state with few artifacts were selected. Spikes and sharp waves were marked in 5-min segments within the previously selected 10-min segment in bipolar montages on a standard EEG display. If fewer than five IEDs were identified, the segment was extended to $10 \mathrm{~min}$. All spikes within polyspikes, sharp waves, and spike-and-wave complexes were counted as individual IEDs. Marking of the segment was visible for both readers; other marks were blinded for the second reader. For each patient, we calculated the mean event rate per minute and then calculated percent changes from baseline period.

Background activity was rated as abnormal if the frequency and reactivity of the background rhythm was not in an age-appropriate range during the awake state. Sleep architecture was rated abnormal if (1) both vertex waves (VW) and K-complexes (KC) were missing, (2) no SS were identified, or (3) no slow-wave sleep (SWS) was noted during the whole night (only in children with VEEG recordings).
Improvement of sleep architecture was recorded if SSeither KC or VW-or SWS that was not present in the first EEG was observed in the second EEG. Normalization was defined as the presence of SS - either VW or KC - and SWS (the latter only for patients with VEEG).

\subsection{Behavior}

For assessment of behavioral problems, the Child Behavior Checklist (CBCL) was applied at baseline and at T1. The preschool version was used in children aged $\leq 5$ years, and the school-age version was used in older children. For the purpose of this study, we included the higher-order scale "internalizing problems," which incorporates the subscales anxious/depressed, withdrawn/depressed, and somatic complaints; the higher-order scale "externalizing problems," which sums up the subscales rule breaking and aggressive behavior; and the total CBCL score, which-in addition to the above-mentioned subscales-incorporates the subscales attention problems, social problems, and thought problems.

\subsection{Statistical Analysis}

The study sample size was based on patient enrolment. We used the statistical software GraphPad Prism, version 9.1.1 (CA, USA; www.graphpad.com) for statistical analyses. Depending on whether or not data were normally distributed (Kolmogorov-Smirnov test), comparisons of two groups were conducted with a paired or unpaired $t$ test or Mann-Whitney $U$ test, and comparisons of more than two groups were conducted with either an analysis of variance or the Kruskal-Wallis test. For correction of post hoc multiple comparisons, a Bonferroni adjustment was applied. For contingency analyses, Fisher's exact test was used. Correlation between the variables was computed using the Pearson method. The threshold of statistical significance was set at $p<0.05$.

We tested three hypotheses: (1) IED rate will decrease significantly from baseline to 3 months of cannabidiol treatment, (2) IED reduction will be higher in patients with $>50 \%$ reduction of seizure frequency, (3) sleep architecture will improve significantly from $\mathrm{T} 0$ to $\mathrm{T} 1$.

\section{Results}

\subsection{Study Population and EEG Type}

Between November 2019 and January 2021, 37 children received cannabidiol for drug-resistant epilepsy. We excluded one patient who discontinued treatment because of side effects and one patient with insufficient EEG data, leaving 35 participants for inclusion in the study. In most 
patients, the number and dose of ASMs was kept stable between $\mathrm{T} 0$ and $\mathrm{T} 1$. In one patient, ethosuximide was discontinued because of gastrointestinal side effects; in another patient, the clobazam dose had to be reduced because of sedation. A VEEG was recorded in 20 patients, sleep was recorded in a routine EEG in three patients, and a routine EEG awake was recorded in 12 patients. Detailed patient characteristics are shown in Table 1.

\subsection{Seizure Reduction and Cannabidiol Dose}

The mean \pm standard deviation (SD) percentage reduction of motor seizures was $54.7 \pm 26.1 \%$ at 3 months. One patient was free of all motor seizures during the 3-month treatment period. No patient reported an increase in countable seizure frequency. A total of 24 patients had a reduction of their motor seizures of $\geq 50 \%$ ("responders"). Mean cannabidiol dose at $\mathrm{T} 1$ was $23.8 \pm 9.6 \mathrm{mg} / \mathrm{kg} / \mathrm{day}$. We recorded seizures during the evaluated night via VEEG in $11 / 20$ patients at T0 (median seizures 5 [range 2-15]) and in 9/20 patients at $\mathrm{T} 1$ (median seizures 2 [range 1-11]). In all cases, the minimum distance of $1 \mathrm{~h}$ between last and/or next seizure and segment for IED evaluation could be maintained. No seizures were recorded in any of the routine EEGs.

\subsection{Interictal Epileptiform Discharges}

Cohen's kappa for IEDs was 0.8 and for background activity/ sleep architecture was 0.94 . After discussion of the nonconcordant events, consensus was reached in all cases. All 35 patients had IEDs in their initial EEG with a mean \pm SD IED rate of $36.8 \pm 27.2$ per minute. The EEG segment was extended to 10 minutes at T0 in only one patient because fewer than five IEDs were observed in the 5-minute segment. The segment was also extended at $\mathrm{T} 1$ in the same patient and in another two patients. The initial IED rate did not differ between patients with sleep EEG recordings ( $n=$ 23 ) and those with awake EEG recordings ( $n=12): 34.1 \pm$ 25.4 versus $42.2 \pm 30.8$, respectively; $p=0.41$. Mean $\pm \mathrm{SD}$ reduction of IED rate from T0 to T1 was $56.3 \pm 23.3 \%$. At T1, the IED rate was significantly lower than at T0 (19.6 \pm 19.5 vs. $36.8 \pm 27.2$, respectively; $p<0.0001$ ), which confirmed hypothesis 1 (Fig. 1). The median percent reduction of IEDs was similar in the sleep and awake states (57.5 \pm 23.5 vs. $54.8 \pm 22.9$, respectively; $p=0.85$ ).

To test hypothesis two, we compared mean percentage IED reductions between responders $(n=24)$ and nonresponders $(n=11)$ to cannabidiol therapy. No significant difference was observed $(61.3 \pm 20.1$ vs. $45.5 \pm 26.9$, respectively; $p=0.08$ ), so hypothesis 2 was not confirmed.

No significant difference was found $(p=0.08)$ in percentage of IED reduction between patients with Dravet syndrome $(n=5)$, LGS $(n=4)$, structural $(n=9)$, genetic other than
Table 1 Patient characteristics $(n=35)$

\begin{tabular}{ll}
\hline Characteristics & Data \\
\hline Age (years) & $10.1 \pm 0.86$ \\
Female sex & $18(51.4)$ \\
Epilepsy type & \\
Dravet syndrome & $5(14.3)$ \\
Lennox-Gastaut syndrome & $4(11.4)$ \\
Structural & $9(25.6)$ \\
Focal cortical dysplasia & $1(2.9)$ \\
Other congenital & $2(5.7)$ \\
Acquired & $6(17.0)$ \\
Genetic other than Dravet syndrome & $12(34.3)$ \\
No known mutation & $6(17.1)$ \\
CDKL5 & $1(2.9)$ \\
Trisomy 21 & $2(5.7)$ \\
Angelman syndrome & $1(2.9)$ \\
Rett syndrome & $2(5.7)$ \\
Metabolic & $1(2.9)$ \\
FIRES & $1(2.9)$ \\
Unknown & $3(8.6)$ \\
Age (years) at diagnosis & $2.3 \pm 0.46$ \\
Discontinued ASMs & $5(2-16)$ \\
Current ASMs & $2(1-3)$ \\
Clobazam comedication & $4(11.4)$ \\
Seizure frequency & \\
Daily & $22(62.8)$ \\
Weekly & $10(28.6)$ \\
Monthly & $3(8.6)$ \\
EEG & $27(77.1)$ \\
Video-EEG & $20(57.1)$ \\
Routine EEG awake & $12(34.3)$ \\
Routine EEG asleep & $3(8.6)$ \\
\hline
\end{tabular}

Data are presented as mean \pm standard deviation, $n(\%)$, or median (range)

$A S M$ antiseizure medication, $C B D$ cannabidiol, EEG electroencephalogram, FIRES febrile infection-related epilepsy syndrome

Dravet syndrome $(n=12)$, and other $(n=5)$ etiologies; see Fig. 2.

To see whether the IED rate at T0, percent seizure reduction, cannabidiol dose, or disease duration were correlated with IED reduction, we calculated a Pearson correlation matrix including these four parameters and corrected for multiple testing. There was a moderate correlation between seizure reduction and IED reduction $(r=0.39, p=0.02)$, a moderate negative correlation between IED rate at T0 and IED reduction $(r=-0.34, p=0.04)$, and a moderate but not significant negative correlation between disease duration 


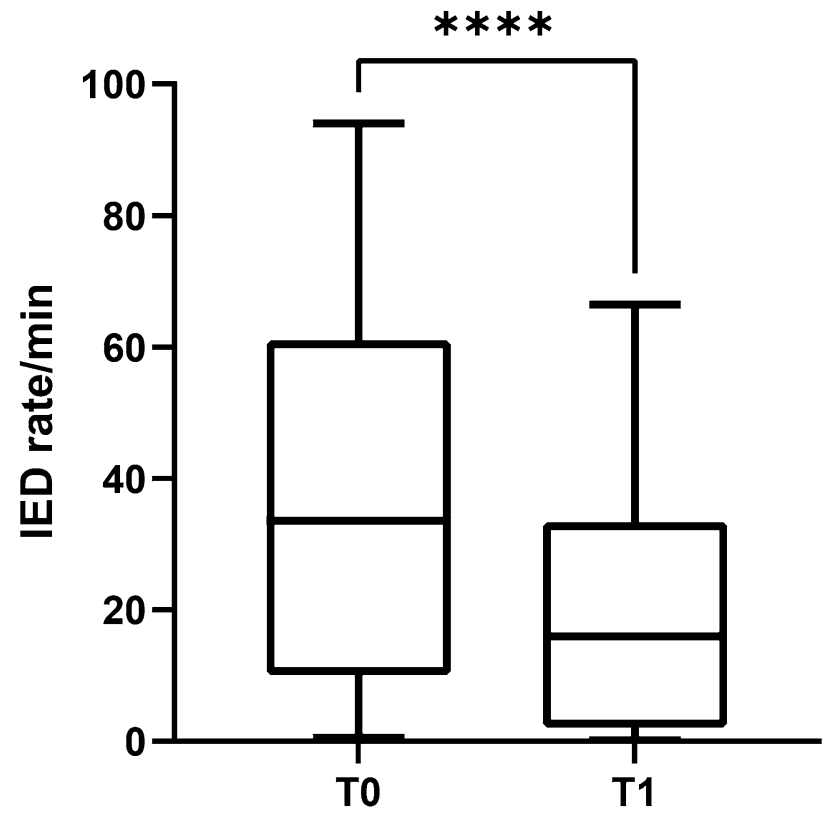

Fig. 1 Rates of interictal epileptiform discharges (IED) per minute at baseline (T0) and after 3 months of cannabidiol therapy (T1). Graph shows median, 25th and 75th percentile, minimum, and maximum. $* * * * p<0.0001$

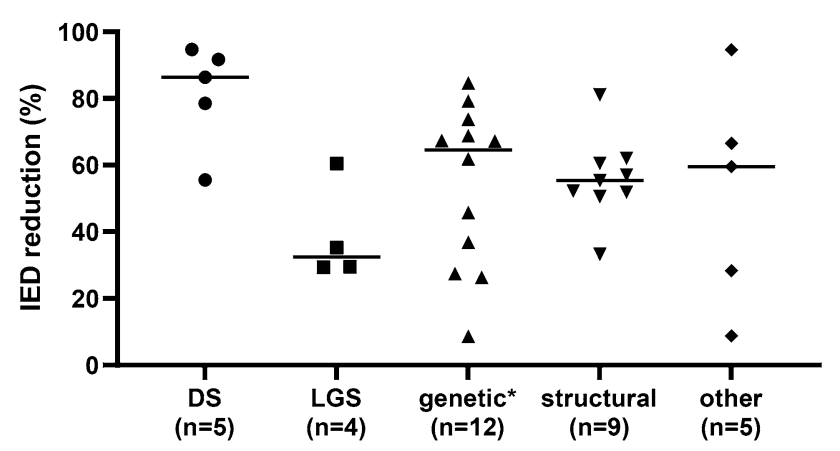

Fig. 2 Reduction of interictal epileptiform discharges (IEDs) at 3 months of cannabidiol therapy in percent from baseline in different groups of epilepsy etiology. DS Dravet syndrome, LGS Lennox-Gastaut syndrome, *Genetic other than Dravet syndrome, 'other' includes febrile infection-related epilepsy syndrome (FIRES; $n=1$ ), metabolic $(n=1)$, unknown $(n=3)$

and IED reduction $(r=-0.32, p=0.06)$. Cannabidiol dose was not significantly correlated with percent IED reduction $(r=-0.20, p=0.25)$.

\subsection{Background Activity and Sleep Architecture}

Initially, sleep architecture was abnormal in 56.5\% $(n=$ 13) of patients in whom sleep was recorded. At T1, sleep had normalized in five patients, but this was not statistically significant $(p=0.11)$. Nevertheless, sleep improved but remained abnormal in six additional patients, so that, in total, sleep had improved in $84.6 \%$ (11/13) of patients. Figure 3 shows the particular sleep elements that led to improvement. Background activity during the awake state was abnormal in $25 \%(n=3)$ of patients in whom no sleep was recorded and improved in two of three. No worsening of sleep architecture or background rhythm was seen.

\subsection{Behavior}

To see whether IED reduction had an effect on behavior, we further analyzed 22 patients for whom CBCL scores were available. This subgroup did not differ in age, cannabidiol dose, seizure reduction, or IED rate at T0 from those in whom the CBCL had not been applied. At T0, in six patients, mean $\pm \mathrm{SD}$ total CBCL scores were within the normal range $(50 \pm 1)$. Total CBCL score improved in 14 (seven of those $>1 \mathrm{SD}$ ), worsened in four (all $<1 \mathrm{SD}$ ), and was unchanged in four patients. The score for internalizing problems (representing depressive and somatic complaints) improved in 14 (three of those $>1 \mathrm{SD}$ ), worsened in five (all $<1 \mathrm{SD}$ ), and was unchanged in three patients. The score for externalizing problems (representing rule breaking and aggressive behavior) improved in 15 (six of those $>1 \mathrm{SD}$ ), worsened in five (all $<1 \mathrm{SD}$ ), and was unchanged in two patients. At a group level, median CBCL scores were significantly lower at $\mathrm{T} 1$ than at baseline: total score 70.6 versus $65.2(p=0.003)$, score of internalizing problems 65.6 versus $59.9(p=0.009)$, and score of externalizing problems 64.1 versus $57.8(p=0.02)$. None of the scores correlated significantly with percent reduction of IED rate from $\mathrm{T} 0$ to $\mathrm{T} 1$ (total score $r=0.39, p=0.07$; internalizing score $r=0.15$, $p=0.5$; externalizing score $r=0.39, p=0.07$ ).

\section{Discussion}

The results of this study indicate that cannabidiol treatment may be effective in reducing IEDs and improving sleep microstructure in children with drug-resistant epilepsy. To the best of our knowledge, this is the first prospective study evaluating the impact of cannabidiol on interictal EEG and sleep structure. With a mean seizure reduction of almost $55 \%$ and a responder rate of $68 \%$, outcomes in this cohort of children with drug-resistant epilepsy of various etiologies were similar to those in other open-label studies $[5,20]$. The mean cannabidiol dose applied in this study was also similar to that in other studies, but the rate of clobazam comedication was lower (11.4\%) [5].

The main focus of ASM development has traditionally been seizure reduction. In recent years, it has been recognized that other outcomes, such as cognitive function or behavior, are similarly important [21]. Cognitive impairment 
A

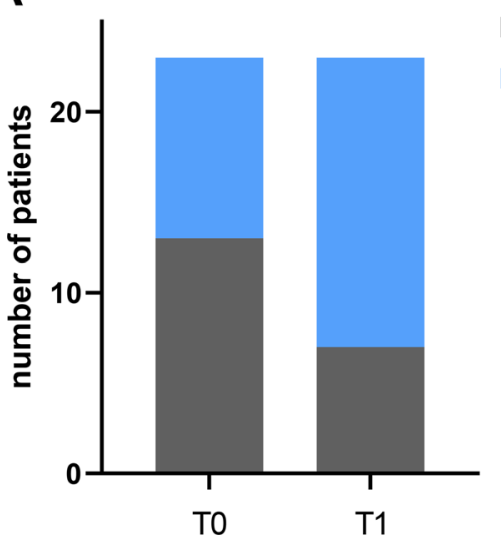

Fig. 3 A Total number of patients with normal and abnormal sleep microstructure at $\mathrm{T} 0$ and $\mathrm{T} 1 ; \mathbf{B}$ number of patients with presence of sleep spindles (SS), vertex waves (VW)/K-complexes (KC), and slow wave sleep (SWS) at baseline (T0) and after 3 months of cannabidiol

is a common comorbidity in children with drug-resistant epilepsy that is considered to have multifactorial origins [22]. However, there is now clear evidence in animals and humans that IEDs can result in cognitive impairment, especially if they are frequent and occur during important periods of brain development [23]. It is also widely accepted that sleep is relevant for memory consolidation and other cognitive processes $[18,24]$ and that IEDs during sleep and sleep disruption in patients with epilepsy have a negative impact on cognitive functioning [19,25]. As for other newly developed ASMs, previous studies of cannabidiol treatment in epilepsy therapy also focused on seizure outcomes. However, preclinical data already suggest that cannabidiol might have additional benefits on comorbidities such as movement disorders, behavioral problems, and memory dysfunction [26]. In humans, the evidence of cognitive evolution during cannabidiol therapy is sparse. A few studies reported some improvement of language and motor skills, alertness, and sleep, but these were based on parental observations rather than standardized tests [27]. In a previous study in children and adults, we showed a slight improvement of selective attention but stable results in other investigated cognitive domains [28]. No studies have provided data to investigate the relationship between IEDs, sleep, and cognitive function during cannabidiol therapy. In this cohort of children with drug-resistant epilepsy of various etiologies, IED rates decreased significantly under add-on therapy with cannabidiol; sleep architecture improved in almost $85 \%$ of cases that were initially abnormal. However, since most of the patients included in this study could not be tested with standardized measures, we could not provide solid data on cognitive function either. Nevertheless, based on the results of this
B

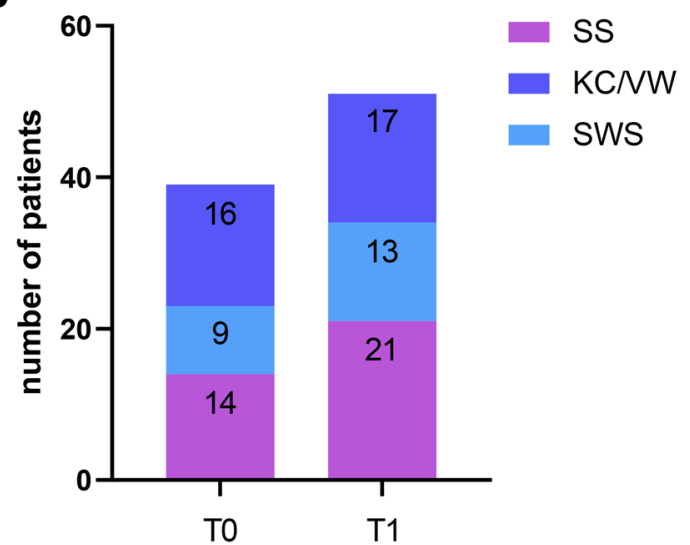

therapy (T1). Total number of patients with sleep recordings $(n=$ 23); SS and VW/KC were rated in all patients, SWS only in patients with whole night recordings $(n=20)$

study, it can be hypothesized that a reduction of IEDs and improved sleep microstructure during cannabidiol therapy might be contributing factors to the potential of cannabidiol to improve cognitive function. It is worth investigating this hypothesis in future studies with larger and more homogeneous cohorts.

Change in behavior was not the main focus of this study, and data on behavioral problems are not available in all patients. Nevertheless, our data suggest that cannabidiol might have a positive influence on behavior overall, and particularly internalizing and externalizing problems. Pressler et al. [29] showed an improvement in behavior in children with epilepsy if IEDs were reduced in frequency or duration. In this study, IED reduction was not significantly correlated with improved CBCL scores; however, the correlation was likely underpowered. As behavior can be analogous to cognitive functioning, it seems worth investigating the influence of cannabidiol-associated IED reductions on behavior in future studies.

The presence or absence of IEDs, the rate of IEDs, and the extent of IED reduction depend on many different factors such as epilepsy type, age, dose and type of medication, level of alertness during EEG, and seizure frequency. In this heterogeneous cohort, a proper statistical comparison of efficacy in IED reduction between different epilepsy types was not possible. Nevertheless, despite small numbers, there might be a trend towards a lower IED reduction in patients with LGS than in those with other epilepsy types. Additionally, a higher IED rate at the beginning, as for patients with LGS, was negatively correlated with IED reduction during treatment. A similar effect was shown for levetiracetam therapy in children with attention-deficit/hyperactivity disorder (with 
and without epilepsy), where children with a higher spikewave index (SWI) showed a smaller SWI reduction [30]. Although IED reduction did not differ between responders and nonresponders, there was a moderate correlation of percent seizure reduction and IED reduction. Previous studies have also shown a correlation between IED reduction and treatment response [14], but the exact relationship between IED rate and disease activity remains unclear. Disease duration trended towards being negatively correlated with IED reduction, but we could not show any correlation between cannabidiol dose and IED reduction. This might be because of the highly variable bioavailability of cannabidiol [31]. On the other hand, it might simply reflect the multifactorial influences on IED reduction, as studies with other ASMs also found no correlation between serum levels and IED rate [32]. Measurement of levels of cannabidiol or its active components were not widely available at the time of this study. For future studies, it would be helpful to correlate both cannabidiol dose and levels and active components with outcome measures. Overall, our data reflect the multifactorial influences on IED rate and reduction.

Strengths of our study include the prospective design and the stable comedication in almost all cases. However, there are also limitations, of which the small sample size and the heterogeneous cohort are the most important. Statistical analyses beyond the predefined hypotheses are limited, and those results are difficult to translate into meaningful clinical correlations. In relation to the heterogeneous epilepsy types included in the study, there was a broad range of IED rates at T0. Since the IED rate at the beginning was negatively correlated with the percent reduction in IEDs at T1, this difference in initial IED rate might have influenced our results. Another limitation was the different EEG modes within the study, with no VEEG being performed in 15 patients and no sleep being recorded in 12 of those. Future studies should aim for a more uniform EEG protocol and examine larger cohorts to enable examination of subgroups with uniform epilepsy types. Inter-rater agreement was high in our study. However, using an automated and standardized EEG analysis might further reduce potential biases, and its use should be considered for future studies.

\section{Conclusion}

Our results strongly suggest the utility of cannabidiol in reducing IEDs and improving sleep microstructure in children with drug-resistant epilepsy. Larger controlled studies are needed to evaluate the clinical relevance of this effect in different epilepsy types.
Acknowledgements KAK and JS were supported by the Berta-Ottenstein-Program for Clinician Scientists from the Faculty of Medicine, University of Freiburg. JJ was supported by the German Research Foundation (DFG; JA 1725/4-1).

\section{Declarations}

Funding Open Access funding enabled and organized by Projekt DEAL. This research received no specific grant from any funding agency in the public, commercial, or nonprofit sector.

Conflict of interest Kerstin A. Klotz has received honoraria for lectures and advice from EISAI, GW Pharmaceuticals, and Zogenix. Jan Schönberger has received an honorarium for a lecture and advice from GW pharmaceuticals. Andreas Schulze-Bonhage has received honoraria for lectures and advice from BIAL, EISAI, Precisis, and UCB. Daniel Grob, Lea Nakamura, Brigitta Metternich, and Julia Jacobs have no conflicts of interest that are directly relevant to the content of this article.

Ethics approval The trial was approved by the ethics committee of the Albert-Ludwigs-University Freiburg (397/17).

Consent to participate All patients or their parents/legal representatives provided written informed consent and assent according to patients' physical and mental capability before trial onset.

Consent for publication Not applicable.

Availability of data and material The dataset supporting the conclusions of the manuscript will be made available by the authors, to any qualified researcher, without breaching participant confidentiality.

Code availability Not applicable.

Author contributions KAK designed and conceptualized the study, played a major role in the acquisition of data, analyzed the data, and drafted the manuscript for intellectual content. DG had a major role in the acquisition of data. JS and LN had a major role in the acquisition of data and revised the manuscript for intellectual content. BM and ASB interpreted the data and revised the manuscript for intellectual content. JJ provided advice in conceptualizing the study, interpreted the data, and revised the manuscript for intellectual content. All authors read and approved the final version of the manuscript and agreed to be accountable for the work.

Open Access This article is licensed under a Creative Commons Attribution-NonCommercial 4.0 International License, which permits any non-commercial use, sharing, adaptation, distribution and reproduction in any medium or format, as long as you give appropriate credit to the original author(s) and the source, provide a link to the Creative Commons licence, and indicate if changes were made. The images or other third party material in this article are included in the article's Creative Commons licence, unless indicated otherwise in a credit line to the material. If material is not included in the article's Creative Commons licence and your intended use is not permitted by statutory regulation or exceeds the permitted use, you will need to obtain permission directly from the copyright holder. To view a copy of this licence, visit http://creativecommons.org/licenses/by-nc/4.0/. 


\section{References}

1. Devinsky O, Cross JH, Wright S. Trial of cannabidiol for drug-resistant seizures in the Dravet Syndrome. N Engl J Med. 2017;377:699-700. https://doi.org/10.1056/NEJMoa1611618.

2. Devinsky O, Patel AD, Cross JH, Villanueva V, Wirrell EC, Privitera $\mathrm{M}$, et al. Effect of cannabidiol on drop seizures in the LennoxGastaut syndrome. N Engl J Med. 2018;378:1888-97. https://doi. org/10.1056/NEJMoa1714631.

3. Thiele EA, Marsh ED, French JA, Mazurkiewicz-Beldzinska M, Benbadis SR, Joshi C, et al. Cannabidiol in patients with seizures associated with Lennox-Gastaut syndrome (GWPCARE4): a randomised, double-blind, placebo-controlled phase 3 trial. Lancet. 2018;391:1085-96. https://doi.org/10.1016/S0140-6736(18) 30136-3.

4. Thiele EA, Bebin EM, Bhathal H, Jansen FE, Kotulska K, Lawson JA, et al. Add-on cannabidiol treatment for drug-resistant seizures in tuberous sclerosis complex: a placebo-controlled randomized clinical trial. JAMA Neurol. 2020;78(3):285-92. https://doi.org/ 10.1001/jamaneurol.2020.4607.

5. Szaflarski JP, Bebin EM, Comi AM, Patel AD, Joshi C, Checketts $\mathrm{D}$, et al. Long-term safety and treatment effects of cannabidiol in children and adults with treatment-resistant epilepsies: expanded access program results. Epilepsia. 2018;59:1540-8. https://doi. org/10.1111/epi.14477.

6. Klotz KA, Grob D, Hirsch M, Metternich B, Schulze-Bonhage A, Jacobs J. Efficacy and tolerance of synthetic cannabidiol for treatment of drug resistant epilepsy. Front Neurol. 2019;10:1313. https://doi.org/10.3389/fneur.2019.01313.

7. Lattanzi S, Brigo F, Cagnetti C, Trinka E, Silvestrini M. Efficacy and safety of adjunctive cannabidiol in patients with Lennox-Gastaut syndrome: a systematic review and metaanalysis. CNS Drugs. 2018;32:905-16. https://doi.org/10.1007/ s40263-018-0558-9.

8. Roebuck AJ, Greba Q, Onofrychuk TJ, McElroy DL, Sandini TM, Zagzoog A, et al. Dissociable changes in spike and wave discharges following exposure to injected cannabinoids and smoked cannabis in Genetic Absence Epilepsy Rats from Strasbourg. Eur J Neurosci. 2020. https://doi.org/10.1111/ejn.15096.

9. Prakash V. Effect of cannabinoids on electroencephalography of a child with Lennox-Gastaut syndrome. J Neurosci Rural Pract. 2020;11:643-5. https://doi.org/10.1055/s-0040-1714329.

10. Kuchenbuch M, D’Onofrio G, Chemaly N, Barcia G, Teng T, Nabbout R. Add-on cannabidiol significantly decreases seizures in 3 patients with SYNGAP1 developmental and epileptic encephalopathy. Epilepsia Open. 2020;5:496-500. https://doi.org/10.1002/ epi4.12411.

11. McCoy B, Wang L, Zak M, Al-Mehmadi S, Kabir N, Alhadid K, et al. A prospective open-label trial of a CBD/THC cannabis oil in Dravet syndrome. Ann Clin Trans1 Neurol. 2018;5:1077-88. https://doi.org/10.1002/acn3.621.

12. Tzadok M, Uliel-Siboni S, Linder I, Kramer U, Epstein O, Menascu S, et al. CBD-enriched medical cannabis for intractable pediatric epilepsy: the current Israeli experience. Seizure. 2016;35:41-4. https://doi.org/10.1016/j.seizure.2016.01.004.

13. Karoly PJ, Freestone DR, Boston R, Grayden DB, Himes D, Leyde $\mathrm{K}$, et al. Interictal spikes and epileptic seizures: their relationship and underlying rhythmicity. Brain. 2016;139:1066-78. https://doi. org/10.1093/brain/aww019.

14. Goncharova II, Alkawadri R, Gaspard N, Duckrow RB, Spencer DD, Hirsch LJ, et al. The relationship between seizures, interictal spikes and antiepileptic drugs. Clin Neurophysiol. 2016;127:3180-6. https://doi.org/10.1016/j.clinph.2016.05.014.
15. Filippini M, Boni A, Giannotta M, Gobbi G. Neuropsychological development in children belonging to BECTS spectrum: longterm effect of epileptiform activity. Epilepsy Behav. 2013;28:504 11. https://doi.org/10.1016/j.yebeh.2013.06.016.

16. Horak PC, Meisenhelter S, Song Y, Testorf ME, Kahana MJ, Viles WD, et al. Interictal epileptiform discharges impair word recall in multiple brain areas. Epilepsia. 2017;58:373-80. https://doi.org/ 10.1111/epi.13633.

17. Ebus SCM, IJff DM, den Boer JT, van Hall MJH, Klinkenberg $\mathrm{S}$, van der Does A, et al. Changes in the frequency of benign focal spikes accompany changes in central information processing speed: a prospective 2-year follow-up study. Epilepsy Behav. 2015;43:8-15. https://doi.org/10.1016/j.yebeh.2014.11.027.

18. Mason GM, Lokhandwala S, Riggins T, Spencer RMC. Sleep and human cognitive development. Sleep Med Rev. 2021;57: 101472. https://doi.org/10.1016/j.smrv.2021.101472.

19. Novak V, Maulisova A, Jezdik P, Benova B, Belohlavkova A, Liby $\mathrm{P}$, et al. Generalized quasiperiodic epileptiform activity in sleep is associated with cognitive impairment in children with drugresistant focal lesional epilepsy. Epilepsia. 2019;60:2263-76. https://doi.org/10.1111/epi.16362.

20. Devinsky O, Marsh E, Friedman D, Thiele E, Laux L, Sullivan $\mathrm{J}$, et al. Cannabidiol in patients with treatment-resistant epilepsy: an open-label interventional trial. Lancet Neurol. 2016;15:270-8. https://doi.org/10.1016/S1474-4422(15)00379-8.

21. Murugupillai R, Ranganathan SS, Wanigasinghe J, Muniyandi $\mathrm{R}$, Arambepola C. Development of outcome criteria to measure effectiveness of antiepileptic therapy in children. Epilepsy Behav. 2018;80:56-60. https://doi.org/10.1016/j.yebeh.2017.12.011.

22. Hermann B, Seidenberg M, Jones J. The neurobehavioural comorbidities of epilepsy: can a natural history be developed? Lancet Neurol. 2008;7:151-60. https://doi.org/10.1016/S1474-4422(08) 70018-8.

23. Holmes GL. EEG abnormalities as a biomarker for cognitive comorbidities in pharmacoresistant epilepsy. Epilepsia. 2013;54(Suppl 2):60-2. https://doi.org/10.1111/epi.12186.

24. Ferini-Strambi L, Galbiati A, Marelli S. Sleep microstructure and memory function. Front Neurol. 2013;4:159. https://doi.org/10. 3389/fneur.2013.00159.

25. Lambert I, Tramoni-Negre E, Lagarde S, Pizzo F, Trebuchon-Da Fonseca A, Bartolomei F, et al. Accelerated long-term forgetting in focal epilepsy: do interictal spikes during sleep matter? Epilepsia. 2021;62:563-9. https://doi.org/10.1111/epi.16823.

26. Patra PH, Barker-Haliski M, White HS, Whalley BJ, Glyn S, Sandhu $\mathrm{H}$, et al. Cannabidiol reduces seizures and associated behavioral comorbidities in a range of animal seizure and epilepsy models. Epilepsia. 2019;60:303-14. https://doi.org/10.1111/epi. 14629.

27. Press CA, Knupp KG, Chapman KE. Parental reporting of response to oral cannabis extracts for treatment of refractory epilepsy. Epilepsy Behav. 2015;45:49-52. https://doi.org/10.1016/j. yebeh.2015.02.043.

28. Metternich B, Wagner K, Geiger MJ, Hirsch M, Schulze-Bonhage A, Klotz KA. Cognitive and behavioral effects of cannabidiol in patients with treatment-resistant epilepsy. Epilepsy Behav. 2021;114: 107558. https://doi.org/10.1016/j.yebeh.2020.107558.

29. Pressler RM, Robinson RO, Wilson GA, Binnie CD. Treatment of interictal epileptiform discharges can improve behavior in children with behavioral problems and epilepsy. J Pediatr. 2005;146:112-7. https://doi.org/10.1016/j.jpeds.2004.08.084.

30. Bakke KA, Larsson PG, Eriksson A-S, Eeg-Olofsson O. Levetiracetam reduces the frequency of interictal epileptiform discharges during NREM sleep in children with ADHD. Eur J 
Paediatr Neurol. 2011;15:532-8. https://doi.org/10.1016/j.ejpn. 2011.04.014.

31. Birnbaum AK, Karanam A, Marino SE, Barkley CM, Remmel RP, Roslawski M, et al. Food effect on pharmacokinetics of cannabidiol oral capsules in adult patients with refractory epilepsy. Epilepsia. 2019;60:1586-92. https://doi.org/10.1111/epi.16093.
32. Stodieck S, Steinhoff BJ, Kolmsee S, van Rijckevorsel K. Effect of levetiracetam in patients with epilepsy and interictal epileptiform discharges. Seizure. 2001;10:583-7. https://doi.org/10.1053/seiz. 2001.0582. 